Pacific Journal of Mathematics

HERMITIAN QUADRATIC FORMS AND HERMITIAN DAVID MORPH 


\title{
HERMITIAN QUADRATIC FORMS AND HERMITIAN MODULAR FORMS
}

\author{
David Mordecai CoHen and H.L. ResnikofF
}

\begin{abstract}
It is shown that if $H$ is a positive definite Hermitian quadratic form in $r$ variables which is even integral over the imaginary quadratic field of discriminant $-d$ and if det $H=2^{r} d^{-r / 2}$, then 4 divides $r$.
\end{abstract}

1. It has long been known that an even integral unimodular positive quadratic form over $\boldsymbol{Z}$ must have rank divisible by 8 ([6], [7]). An interesting consequence of this fact is that if $s>r$, then the $C$-linear space of Siegel modular forms of degree $s$ and weight $r / 2$ is $\{0\}$ unless 8 divides $r([12])$.

The purpose of the present note is to prove an analogous theorem about Hermitian quadratic forms over imaginary quadratic number fields, and to draw the corresponding conclusions about certain $\boldsymbol{C}$-linear spaces of Hermitian modular forms.

Let $K$ be the imaginary quadratic number field of discriminant $-d$ with ring of integers $\mathscr{O}=\boldsymbol{Z}+\boldsymbol{Z} \omega$. Let $\mathfrak{A}_{i}, \mathfrak{B}_{i}, \mathfrak{C}_{i}$ be fractional ideals and $L=\mathfrak{A}_{1} x_{1}+\cdots+\mathfrak{A}_{r} x_{r}$ a finitely generated $\mathcal{O}^{\prime}$-module. Then the ideal class of $\mathfrak{A}=\mathfrak{A}_{1} \cdots \mathfrak{A}_{r}$ is an invariant of $L$. Let $H$ : $L \times L \rightarrow K$ be a Hermitian form of rank $r$. We define the determinant of $H$ by $|H|=\operatorname{Norm}_{K / Q}(\mathfrak{U})\left|H\left(x_{i}, x_{j}\right)\right|$. Then $|H|$ depends only on $H$ and $L$ [8, page 229].

Associated to $H$ are the ideal scale $(H)=\{H(v, w) \mid v, w \in L\}$ and the ideal $\operatorname{norm}(H)$ generated by $\{H(v, v) \mid v \in L\}$. Since $H(v, w)=$ $\overline{H(w, v)}$, norm $(H) \subset \boldsymbol{Q} . \quad H$ is called positive definite if norm $(H) \subset$ $\boldsymbol{Q}^{+}$, and even integral if norm $(H) \subset 2 \boldsymbol{Z}$. Since $\operatorname{trace}_{K / Q}(H(v, w))=$ $H(v, w)+H(w, v)=H(v+w, v+w)-H(v, v)-H(w, w), \operatorname{trace}_{K / Q}$ (scale $(H)) \subset \operatorname{norm}(H) \subset$ scale $(H)$ and an even integral Hermitian form $H$ need not have integral scale.

THEOREM 1. If $H$ is a positive, even integral Hermitian form of rank $r$, and $|H|=2^{r} d^{-r / 2}$, then 4 divides $r$.

The proof of this theorem consists of the assignment to $H$ of the $Z$-bilinear form $B: L \times L \rightarrow \boldsymbol{Q}$ defined by $B(v, w)=(H(v, w)+$ $H(w, v)) / 2$. If $H$ is positive, even integral and of determinant $|H|=$ $2^{r} d^{-r / 2}$, then $B$ is positive even integral and unimodular. Since $\operatorname{dim}_{z} L=2 r$, the assertion follows immediately from the corresponding classical fact. 
Once the assignment $H \mapsto Q$ is specified, the desired properties can be obtained by direct algebraic considerations. However, in view of our intended application and because it provides a natural organization and thematic coherence for the various details of the proof, we prefer to cloak the algebra in the garb of the theory of theta functions. For convenience, we give the proof only in the case when $L=\mathscr{O} x_{1}+\cdots+\mathscr{O} x_{r}$ is a free $\mathscr{O}$-module. Then $|H|=$ $\left|H\left(x_{i}, x_{j}\right)\right|$ and without the risk of confusion we can let $H$ also denote the Hermitian matrix $\left(H\left(x_{i}, x_{j}\right)\right)$.

2. Denote by $\mathfrak{X}_{\mathscr{C}}^{s}$ the set of $s$-rowed Hermitian matrices considered as a real vector space. The subset $\mathfrak{Y}_{\mathscr{C}}^{s}$ of positive Hermitian matrices is a strictly convex open cone in $\mathfrak{S}_{\mathscr{O}}^{s}$. The subset $\mathfrak{Z}_{\mathscr{X}}^{*}=$ $\mathfrak{X}_{\mathscr{C}}^{s}+i \mathfrak{Y}_{\mathscr{X}}^{s}$ of the complexification of $\mathfrak{X}_{\mathscr{O}}^{s}$ is biholomorphically equivalent to a bounded symmetric domain, and is called the Hermitian half space of rank s. The group of biholomorphic automorphisms of $\beta_{\mathscr{K}}^{s}$ consists of the mappings

$$
Z \mapsto(A Z+B)(C Z+D)^{-1}
$$

where the complex matrix $M=\left(\begin{array}{ll}A & B \\ C & D\end{array}\right)$ satisfies $\bar{M}^{\prime}\left(\begin{array}{rr}0 & \mathbf{1} \\ -1 & 0\end{array}\right) M=\left(\begin{array}{rr}0 & \mathbf{1} \\ -1 & 0\end{array}\right)$. The Hermitian modular group $\Gamma_{\mathscr{O}}^{s}(K)$ of rank s associated with $K$ is the subgroup of mappings of the form (1) such that the entries of $M$ belong to $O$. A holomorphic function $\varphi: B_{\mathscr{C}}^{s} \rightarrow C$ is a Hermitian modular form for $K$ of weight $w$ if $\varphi(A Z+B)(C Z+D)^{-1}=$ $\varepsilon|C Z+D|^{w} \varphi(Z)$ for all mappings in $\Gamma_{\%}^{s}(K)$, where $\varepsilon$ is a certain root of 1 (see [1], [2]).

There are theta functions naturally associated with $3_{\mathscr{C}}^{s}$. Let $H$ denote a positive Hermitian quadratic form in $r$ variables and let $\&$ stand for a lattice in $C^{r \times s}$ considered as a real vector space. Then the theta function on $8^{s}$ associated with $H$ and $\&$ is (cp. [11]):

$$
\Theta_{\Omega}(Z, H)=(\operatorname{vol} \Omega)^{1 / 2} \sum_{N \in \Omega} \exp i \pi \sigma\left(Z \bar{N}^{\prime} H N\right),
$$

where vol $\&$ denotes the volume of the lattice $\&$ with respect to a Haar measure on $C^{r s}=R^{2 r s}$, and $\sigma$ stands for matrix trace. This theta function satisfies the following basic functional equation, whose proof consists of a standard application of the Poisson summation formula ([11], Theorem 2.2 with $z_{1 / 2}=0$ ):

$$
\Theta_{\hat{\imath}}\left(-Z^{-1}, H^{-1}\right)=|-i Z|^{r}|H|^{s} \Theta_{\varepsilon}(Z, H)
$$

where $\hat{\mathcal{L}}$ is the dual lattice defined by

$$
\widehat{\Omega}=\left\{\widehat{N} \in \mathbb{C}^{r \times s}: \sigma(\hat{N} N) \in \mathbb{Z} \text { for all } N \in \mathbb{R}\right\} \text {. }
$$


We will determine necessary and sufficient conditions on $H$ such that $\Theta_{\mathcal{Q}}(Z, H)$ be a Hermitian modular form for $\Gamma_{\mathscr{H}}^{s}(K)$ of weight $r$ if the lattice $\mathbb{R}=(\mathscr{O})^{r \times s}$.

It will suffice to consider the behavior of $\Theta_{\varepsilon}(Z, H)$ with respect to the following set of generators of $\Gamma_{\mathscr{H}}^{s}(K)$ :

(1) Translations: $Z \mapsto Z+B$, where $B$ is a Hermitian matrix over $\mathcal{O}$;

(2) Unimodular transformations: $Z \mapsto \bar{U}^{\prime} Z U$, where $U$ is a unimodular matrix over $\mathcal{O}$, and

(3) Partial involutions: $Z \mapsto J_{s_{1}} Z$, where $s_{1}$ is an integer, $0<s_{1} \leqq s$, and the transformation $J_{s_{1}}$ corresponds to the Hermitian modular matrix

$$
\left(\begin{array}{cc|cc}
0 & 0 & -\mathbf{I}_{s_{1}} & 0 \\
0 & \mathbf{1}_{s_{2}} & 0 & 0 \\
\hline \mathbf{1}_{s_{1}} & 0 & 0 & 0 \\
0 & 0 & 0 & \mathbf{1}_{s_{2}}
\end{array}\right)=\left(\begin{array}{cc}
A & B \\
C & D
\end{array}\right) ;
$$

$s_{2}=s-s_{1}$, and $\mathbf{1}_{k}$ denotes the unit matrix of rank equal to $k$.

If $s_{1}=s$, then the partial involution $J_{s}$ is just the involution $Z \mapsto-Z^{-1}$.

First observe that a $\Gamma_{\mathscr{Z}}^{s}(K)$-modular form is invariant under the translation $Z \mapsto Z+B$ where $B$ is a Hermitian matrix over $\mathcal{O}$. It follows that $H$ must be even integral over $O$.

The function $\Theta_{\Omega}(Z, H)$, with $H$ even integral over $\mathcal{O}$, is invariant under the unimodular mappings $Z \mapsto \bar{U}^{\prime} Z U$, where $U$ is a unimodular matrix over $\mathcal{O}$ ([11], Theorem 2.3); hence, it remains to consider the constraints imposed by the partial involutions in conjunction with the functional equation (3).

First we will consider involution $Z \mapsto-Z^{-1}$, and determine the conditions it imposes on $H$. Then we will show that if $H$ satisfies the conditions imposed by the translations and the involution, then it automatically satisfies the constrains imposed by the various partial involutions.

The lattice $\hat{\&}$ can be easily expressed in terms of $\mathfrak{L}$. In fact, since the different of $K$ is the principal ideal generated by $\sqrt{-d}$, one finds $\hat{\Omega}=(2 / \sqrt{-d}) \mathbb{R}$, and consequently $\Theta_{\hat{\Omega}}$ can be expressed in terms of $\Theta_{\Omega}$ by

$$
\Theta_{\hat{\imath}}(Z, H)=\frac{1}{\operatorname{vol} \mathbb{Q}} \Theta_{\Omega}\left(Z, \frac{4 H}{d}\right) ;
$$

here we have used $(\operatorname{vol} \mathfrak{R})(\operatorname{vol} \hat{\mathfrak{L}})=1$. Then $(3)$ becomes 


$$
\Theta_{\Omega}\left(-Z^{-1}, \frac{4 H^{-1}}{d}\right)=|-i Z|^{r}|H|^{s}(\operatorname{vol} \Omega) \Theta_{\Omega}(Z, H) .
$$

If $\Theta_{\mathcal{\Omega}}(Z, H)$ is a $\Gamma_{\mathscr{S}}^{s}(K)$-modular form of weight $r$, then in particular

$$
\Theta_{\AA}\left(-Z^{-1}, H\right)=|-i Z|^{r} \Theta_{\mathcal{\Omega}}(Z, H) \text {. }
$$

Using (7) to rewrite the right side as a function of $-Z^{-1}$, and then replacing $-Z^{-1}$ by $Z$, obtain

$$
\Theta_{\Omega}\left(Z, \frac{4 H^{-1}}{d}\right)=\operatorname{vol} \&|H|^{s} \Theta_{\mathcal{R}}(Z, H) \text {. }
$$

Now write $Z=X+i Y$, with $X$ and $Y$ Hermitian, and compute the limit as the eigenvalues of $Y$ approach infinity (and $X$ remains in some compact set); only the constant term ( $\operatorname{vol} \&)^{1 / 2}$ survives in the theta function (because $Y$ and $H$ are positive) as one immediately sees from the defining equation (2). Therefore $|H|^{s}($ vol $\&)=1$. Since $\mathbb{L}=\mathcal{O}^{r \times s}$, vol $\mathbb{L}=(\sqrt{ } \bar{d} / 2)^{r s}$ and hence

$$
|H\rangle=2^{r} d^{-r / 2} \text {. }
$$

Using this condition, the left side of (7) can be rewritten. Abbreviate $\widetilde{H}=(\sqrt{-d} / 2) H$; then $|\widetilde{H}|=1$ and

$$
\Theta_{\imath}\left(-Z^{-1}, \frac{4 H^{-1}}{d}\right)=\Theta_{\imath}\left(-Z^{-1}, 4 \tilde{\tilde{H}}^{\prime} \frac{H^{-1}}{d} \tilde{H}\right)
$$

(since $\widetilde{H}$ is integral and induces a mere basis change of $\mathbb{R}$ )

$$
=\Theta_{8}\left(-Z^{-1}, H\right) \text {. }
$$

Hence (7) reduces to the functional equation for a $\Gamma_{\mathscr{S}}^{s}(K)$-modular form of weight $r$ under the involution $Z \mapsto-Z^{-1}$.

Now we will show that if $H$ is even integral over $\mathcal{O}$ and $|H|=2^{r} d^{-r / 2}$, then $\Theta_{\Omega}(Z, H)$ is a $\Gamma_{\mho \mathscr{J}}^{s}(K)$-modular form of weight $r$. It is only necessary to verify that $\Theta_{\Omega}(Z, H)$ satisfies the appropriate functional equation for the partial involutions since the other classes of generators of $\Gamma_{\mathscr{H}}^{s}(K)$ have already been considered.

Let $0<s_{1} \leqq s$, let $J_{s_{1}}$ denote the corresponding partial involution with matrix given by (5), and write

$$
Z=\left(\begin{array}{cc}
Z_{1} & Z_{12} \\
Z_{21} & Z_{2}
\end{array}\right)
$$

with $\operatorname{rank} Z_{1}=s_{1}$; observe that $Z_{21}$ is determined by $Z_{12}$. Then

$$
J_{s_{1}} Z=\left(\begin{array}{cc}
-Z_{1}^{-1} & Z_{1}^{-1} Z_{12} \\
Z_{21} Z_{1}^{-1} & Z_{2}-Z_{21} Z_{1}^{-1} Z_{12}
\end{array}\right)
$$


In order to express $\Theta_{\mathcal{Q}}\left(J_{s_{1}} Z, H\right)$ in terms of $\Theta_{\Omega}(Z, H)$, we will expand $\Theta_{2}(Z, H)$ relative to $Z_{2}$. Let $s_{2}=s-s_{1}$ and decompose $\mathbb{Q}=$ $\mathfrak{R}_{1} \oplus \mathfrak{R}_{2}, \mathfrak{R}_{i}=\mathcal{O}^{\mathrm{r} \times s_{i}}$. Write $\mathfrak{R} \ni N=\left(\begin{array}{l}N_{1} \\ N_{2}\end{array}\right)$ with $N_{i} \in \mathfrak{R}_{i}$ and introduce the theta function $\Theta_{\Omega_{1}}\left(Z_{1}, Z_{12}, H\right)$ by

$$
\begin{aligned}
\Theta_{\Omega_{1}}\left(Z_{1}, Z_{12}, H\right)= & \left(\operatorname{vol} \Re_{1}\right)^{1 / 2} \sum_{N_{1} \in \Omega_{1}} \exp i \pi \sigma\left(N_{1} Z_{1} \bar{N}_{1}^{\prime} H\right. \\
& \left.+N_{1} Z_{12} H+Z_{21} \bar{N}_{1}^{\prime} H\right) .
\end{aligned}
$$

Then, noting that $\operatorname{vol} \&=\left(\operatorname{vol} \&_{1}\right)\left(\operatorname{vol} \Omega_{2}\right)$, obtain

$$
\Theta_{8}(Z, H)=\left(\operatorname{vol} \Re_{2}\right)^{1 / 2} \sum_{N_{2} \in \AA_{2}} \Theta_{\Omega_{1}}\left(Z_{1}, Z_{12} \bar{N}_{2}^{\prime}, H\right) \exp i \pi \sigma\left(Z_{2} \bar{N}_{2}^{\prime} H N_{2}\right)
$$

By (9),

$$
\begin{aligned}
& \Theta_{\mathfrak{R}}\left(J_{s_{1}} Z, H\right) \\
& =\left(\operatorname{vol} \Omega_{2}\right)^{1 / 2} \sum_{N_{2} \in \varepsilon_{2}} \Theta_{\Omega_{1}}\left(-Z_{1}^{-1}, Z_{1}^{-1} Z_{12} \bar{N}_{2}^{\prime}, H\right) \\
& \times \exp -i \pi \sigma\left(N_{2} Z_{21} Z_{1}^{-1} Z_{12} \bar{N}_{2}^{\prime}\right) \exp i \pi \sigma\left(Z_{2} \bar{N}_{2}^{\prime} H N_{2}\right) \text {. }
\end{aligned}
$$

The theta function $\Theta_{\Omega_{1}}\left(Z_{1}, Z_{12}, H\right)$ satisfies a transformation formula analogous to (3) which will enable us to rewrite the right hand side of (12). In fact, ([10], Theorem 2.2) asserts

$$
\begin{aligned}
& \Theta_{\hat{\iota}_{1}}\left(-Z^{-1}, Z_{1}^{-1} Z_{12} H, H^{-1}\right) \\
& \quad=\left|-i Z_{1}\right|^{r}|H|^{s} \exp i \pi \sigma\left(Z_{21} Z_{1}^{-1} Z_{12} H\right) \Theta_{\Omega_{1}}\left(Z_{1}, Z_{12}, H\right) .
\end{aligned}
$$

Since $\hat{\mathfrak{\imath}}_{1}=(2 / \sqrt{-d}) \mathfrak{Q}_{1}$,

$$
\Theta_{\hat{\mathfrak{s}}_{1}}\left(Z_{1}, Z_{12}, H\right)=\frac{1}{\operatorname{vol} \mathfrak{\Omega}_{1}} \Theta_{\mathfrak{\Omega}_{1}}\left(Z_{1}, \sqrt{-\overline{-d}} Z_{12}, \frac{4 H}{d}\right)
$$

(cp. (6)), whence (13) can be written

$$
\begin{aligned}
& \Theta_{\mathcal{\Omega}_{1}}\left(-Z_{1}^{-1},-\frac{\sqrt{-d}}{2} Z_{1}^{-1} Z_{12} H, \frac{4 H^{-1}}{d}\right) \\
& \quad=\left|-i Z_{1}\right|^{r} \operatorname{vol} \Omega_{1}|H|^{s_{1}} \exp i \pi \sigma\left(Z_{21} Z_{1}^{-1} Z_{12} H\right) \Theta_{\Omega_{1}}\left(Z_{1}, Z_{12}, H\right) .
\end{aligned}
$$

We have assumed that $H$ is even integral over $\mathscr{O}$ and $|H|=$ $2^{r} d^{-r / 2}$; therefore, with $\tilde{H}=(\sqrt{-d} / 2) H$ as before, and replacing $N_{1}$ by $\tilde{H} N_{1}$, find

$$
\Theta_{\Omega_{1}}\left(Z_{1},-\frac{\sqrt{-d}}{2} Z_{1}^{-1} Z_{12} H, \frac{4 H^{-1}}{d}\right)=\Theta_{\Omega_{1}}\left(Z_{1}, Z_{1}^{-1} Z_{12}, H\right) .
$$

Recalling that vol $\mathfrak{1}_{1}=\left(2^{r} d^{-r / 2}\right)^{s_{1}}$, substitution in (14) yields 


$$
\begin{aligned}
& \Theta_{\varepsilon_{1}}\left(-Z_{1}^{-1}, Z_{1}^{-1} Z_{12}, H\right) \\
& \quad=\left|-i Z_{1}\right|^{r} \exp i \pi \sigma\left(Z_{21} Z_{1}^{-1} Z_{12} H\right) \Theta_{\varepsilon_{1}}\left(Z_{1}, Z_{12}, H\right) .
\end{aligned}
$$

Now write $Z_{12} \bar{N}_{2}^{\prime}$ in place of $Z_{12}$ and substitute (15) in (12) to obtain

$$
\begin{gathered}
\Theta_{\Omega}\left(J_{s_{1}} Z, H\right)=\left|-i Z_{1}\right|^{r}\left(\operatorname{vol} \Omega_{2}\right)^{1 / 2} \sum_{N_{2} \in \Omega_{2}} \Theta_{\Omega_{1}}\left(Z_{1}, Z_{12} \bar{N}_{2}^{\prime}, H\right) \\
\times \exp i \pi \sigma\left(Z_{2} \bar{N}_{2}^{\prime} H N_{2}\right)=\left|-i Z_{1}\right|^{r} \Theta_{\Omega}(Z, H) .
\end{gathered}
$$

Since $|C Z+D|=\left|\begin{array}{cc}Z_{1} & Z_{12} \\ 0 & \mathbf{1}_{s_{2}}\end{array}\right|=\left|Z_{1}\right|$ for the modular transformation $J_{s_{1}}$, (16) shows that $\Theta_{\Omega}(Z, H)$ is a modular form of weight $r$ with a multiplier system $\varepsilon$ which equals 1 on the translations and unimodular transformations in $\Gamma_{\mathscr{H}}^{s}(K)$, and equals $(-i)^{r s_{1}}$ on the partial involution $J_{s_{1}}$.

The Siegel half space of rank $s$, denoted $3_{S}^{s}$, is the subspace of $3_{\mathscr{C}}^{s}$ which consists of symmetric complex matrices. The Siegel modular group $\Gamma_{\mathscr{S}}^{s}$ of rank $s$ is the subgroup of $\Gamma_{\mathscr{F}}^{s}(K)$ which consists of those mappings whose corresponding matrices $\left(\begin{array}{ll}A & B \\ C & D\end{array}\right)$ are real. It follows that the restriction of a $\Gamma_{\mathscr{O}}^{s}(K)$-modular form of weight $r$ to $B_{\mathscr{S}}^{s}$ is a $\Gamma_{\mathscr{S}}^{s}$-modular form of weight $r$ ("Siegel" modular form).

In particular, if $\mathbb{L}=(\mathscr{O})^{r \times s}, H$ is even integral over $\mathcal{O}$, and $|H|=2^{r} d^{-r / 2}$, then $\left.\Theta_{\Omega}(Z, H)\right|_{8_{S}^{8}}$ is a Siegel modular form of weight $r$. We can identify this form.

If $Q$ is the matrix of an even integral unimodular quadratic form in $2 r$ variables over $Z$, and if $Z \in B_{\mathscr{S}}^{s}$, then the theta function

$$
\theta(z, Q)=\sum_{n \in Z^{2 r \times s}} \exp i \pi \sigma\left(z n^{\prime} Q n\right)
$$

is a Siegel modular form of weight $r$. In particular, it satisfies the relation

$$
\theta\left(-z^{-1}, Q\right)=|-i z|^{r} \theta(z, Q) .
$$

Set $\omega=-(d+\sqrt{-d}) / 2$; then $\{1, \omega\}$ is a $Z$-basis for $\mathscr{O}$, and the elements of $\mathbb{R}=(\mathscr{O})^{r \times s}$ can be expressed in the form $N=N_{1}+\omega N_{2}$ where $N_{i} \in Z^{r \times s}$. If $Z$ is restricted to $Z_{\mathscr{S}}^{s}$, one obtains

$$
\Theta_{\AA}(z, H)=(\operatorname{vol} \Omega)^{1 / 2} \theta(z, Q(H))
$$

where

$$
Q(H)=\frac{1}{2}\left(\begin{array}{cc}
H+\bar{H} & \omega H+\bar{\omega} \bar{H} \\
\omega H+\bar{\omega} \bar{H} & \omega \bar{\omega}(H+\bar{H})
\end{array}\right) .
$$

Since the restriction of $\Theta_{g}$ to $B_{\mathscr{S}}^{s}$ is a Siegel modular form, it automatically follows that $Q(H)$ is positive, even integral over $\boldsymbol{Z}$, 
and $|Q(H)|=1$. But these conditions entail that 8 divides the rank of $Q(H)$, i.e., 4 divides $r$, which completes the proof of Theorem 1 .

3. Theorem 1 gives necessary conditions for the existence of positive, even integral Hermitian forms $H$ of rank $r$ with determinant $|H|=2^{r} d^{-r / 2}$. In fact, these conditions are sufficient as the following theorem implies.

THEOREM 2. There exists a positive Hermitian matrix $H$ of rank $4,|H|=16 / d^{2}$, which is even integral over 0 .

In modern terminology, we will show that there exists a Hermitian form $H: O^{4} \times O^{4} \rightarrow K$ such that norm $(H) \subset 2 Z^{+}$and $|H|=$ $16 / d^{2}$.

Our construction is based on the following lemma of Jacobson [5]. See also Gerstein [3] for a similar construction.

Lemma (Jacobson). If $K_{\mathfrak{p}}$ is a local field, then any two regular $n$-ary Hermitian spaces over $K_{*}$ with the same determinant are isometric.

$$
\text { Let } \begin{aligned}
L^{\prime} & =\left\langle v_{1}\right\rangle \perp\left\langle v_{2}\right\rangle \perp\left\langle v_{3}\right\rangle \perp\left\langle v_{4}\right\rangle \\
& =\langle 2\rangle \perp\langle 2\rangle \perp\langle 2 / d\rangle \perp\langle 2 / d\rangle .
\end{aligned}
$$

Then $\left|L^{\prime}\right|=16 / d^{2}$ and norm $\left(L^{\prime}\right) \subset(2 / d) Z$. At all primes $\mathfrak{S} / d$ we will use Jacobson's lemma to change $L^{\prime}$ to $L$ so that $L$ will be even integral. Let $p \boldsymbol{Z}=\mathfrak{P} \cap \boldsymbol{Z}$. Then $p \mathcal{O}=\mathfrak{F}^{2}$ and $\mathfrak{P}$ is the only integral with $N(\mathfrak{B})=p$.

For all $\mathfrak{s} \mid d$, let

$$
L_{\mathfrak{\beta}}=\left(\begin{array}{cc}
0 & 2 / \sqrt{-d} \\
-2 / \sqrt{-d} & 0
\end{array}\right) \perp\left(\begin{array}{cc}
0 & 2 / \sqrt{-d} \\
-2 / \sqrt{-d} & 0
\end{array}\right) .
$$

Then $L_{\mathfrak{\beta}}$ is a Hermitian form over $\mathcal{O}_{\Re},\left|L_{\Re}\right|=16 / d^{2}$, and norm $\left(L_{\mathfrak{p}}\right) \subset 2 \boldsymbol{Z}_{p}$. Since $\left|L_{\mathfrak{p}}\right|=\left|L^{\prime}\right|$, by Jacobson's lemma, the Hermitian spaces $L_{\mathfrak{\beta}} \otimes \mathcal{O}_{\mathfrak{\beta}} K_{\mathfrak{\beta}}$ and $L^{\prime} \otimes K_{\mathfrak{\beta}}$ are isometric, and there exists a lattice in $L^{\prime} \otimes K_{\mathfrak{p}}$ which is isometric to $L_{\Re}$. Call that lattice $L_{\Re}$. We have a matrix $T_{\Re}$ with $\Re$-adic coefficients such that

$$
L_{\Re}=T_{\Re} v_{1}+T_{\Re} v_{2}+T_{\Re} v_{3}+T_{\Re} v_{4} \text {. }
$$

For some large integer $n, \mathfrak{S}^{n} L^{\prime} \subset L_{\Re}$. Therefore if $T$ is a matrix with coefficients in $K$ which is close to $T_{\Re}$ in the $\$$-adic topology, then $T\left(L^{\prime}\right) \otimes \mathcal{O}_{\Re}=L_{\Re}$. Since there are only a finite number of prime ideals $\mathfrak{P} \mid d$, we can use the Chinese Remainder Theorem to construct a matrix $T$, with coefficients in $K$, which is 
sufficiently close to $T_{\mathfrak{\beta}}$ for all $\mathfrak{P} \mid d$. Let $M=T\left(L^{\prime}\right)$. Then $\forall \mathfrak{P} \mid d$, $M \otimes \mathscr{O}_{\Re}=L_{\Re}$.

Using the invariant factor theorem [8, page 214], we can find a basis $x_{1}, x_{2}, x_{3}$, and $x_{4}$ for $V=L^{\prime} \otimes K=M \otimes K$ such that

$$
\begin{aligned}
& L^{\prime}=\mathfrak{A}_{1} x_{1}+\mathfrak{A}_{2} x_{2}+\mathfrak{A}_{3} x_{3}+\mathfrak{A}_{4} x_{4} \\
& M=\mathfrak{B}_{1} x_{1}+\mathfrak{B}_{2} x_{2}+\mathfrak{B}_{3} x_{3}+\mathfrak{B}_{4} x_{4} .
\end{aligned}
$$

Since $L^{\prime}$ is a free $\mathcal{O}^{- \text {module, }} \mathfrak{A}=\mathfrak{A}_{1} \mathfrak{A}_{2} \mathfrak{A}_{3} \mathfrak{A}_{4}$ is a principal ideal. Let $\mathfrak{E}_{i}$ be the ideal such that $\operatorname{Ord}_{\mathfrak{P}^{\prime}}\left(\mathfrak{E}_{i}\right)=\operatorname{ord}_{\mathfrak{F}^{\prime}}\left(\mathfrak{A}_{i}\right) \forall \mathfrak{P}^{\prime} \nmid d$, and $\operatorname{Ord}_{\mathfrak{P}}\left(\mathbb{E}_{i}\right)=$ $\operatorname{Ord}_{\mathfrak{R}}\left(\mathfrak{B}_{i}\right) \forall \Re \mid d$. Let $\mathbb{E}=\mathfrak{C}_{1} \mathfrak{C}_{2} \mathfrak{C}_{3} \mathbb{E}_{4}$ and

$$
L=\mathbb{夭}_{1} x_{1}+\mathfrak{\mho}_{2} x_{2}+\mathbb{F}_{3} x_{3}+\mathbb{\mho}_{4} x_{4} .
$$

Then $L \otimes \mathcal{O}_{\Re}=M \otimes \mathcal{O}_{\Re}=L_{\Re} \forall \mathfrak{P} \mid d$, and $L \otimes \mathcal{O}_{\Re^{\prime}}=L^{\prime} \otimes \mathcal{O}_{\Re^{\prime}}, \mathscr{P}^{\prime} \forall \mathfrak{P}^{\prime} \mid d$. Therefore $|L|=16 / d^{2}$ and norm $(L) \subset 2 Z$.

Now $\left|L^{\prime}\right|=N(\mathfrak{A})\left|H\left(x_{i}, x_{j}\right)\right|$ and $|L|=N(\mathbb{E})\left|H\left(x_{i}, x_{j}\right)\right|$. Since $|L|=\left|L^{\prime}\right|, N(\mathfrak{A} / \mathfrak{E})=1$. But $\mathfrak{A}$ and $\mathfrak{E}$ differ only at the ramified primes. Let $\mathfrak{U} / \mathbb{C}=\mathfrak{P}_{1}^{e_{1}} \cdots \mathfrak{P}_{m}^{e_{m}}$ where the $\mathfrak{P}_{i}$ are the different ramified primes, and $p_{i}=N\left(\mathfrak{P}_{i}\right)$ are distinct prime numbers. Then $N(\mathfrak{A} / \mathfrak{C})=1=p_{1}^{e_{1}} \cdots p_{m}^{e_{m}}$ and all $e_{i}=0$. Therefore $\mathfrak{A}=\mathfrak{C}$. Since $L^{\prime}$ is a free $\mathscr{O}$-module, $\mathfrak{A}=\mathfrak{C}$ is principal, and $L$ is free.

For $K=\boldsymbol{Q}(\sqrt{-1})$,

$$
I=\left(\begin{array}{cccc}
2 & -i & -i & 1 \\
i & 2 & 1 & i \\
i & 1 & 2 & 1 \\
1 & -i & 1 & 2
\end{array}\right), \quad i=\sqrt{-1}
$$

is even integral over $Z[\sqrt{-1}]$ and $|I|=1$, cp. Iyanaga [4].

One can show that the set of even integral Hermitian forms with determinant $16 / d^{2}$ forms a genus. But its class number remains unknown; in view of [9], it seems unlikely that it is 1.

4. The results of the previous sections can be applied to the theory of Hermitian modular forms. Since, for $s>4$. $\left(\Gamma_{\mathscr{Z}}^{s}(K), 4\right)$ is spanned by theta functions associated with Hermitian forms of the type described in Theorem 1, Theorem 2 implies:

THEOREM 3. (a) If $s>4$, then $\left(\Gamma_{\mathscr{F}}^{s}(K), 4\right)$ is spanned by $\Theta_{\beta}(Z, L)$, where $\mathbb{L}=\mathcal{O}^{4 \times s}$ and $L$ runs through representatives of the $\mathcal{O}_{- \text {-equi- }}$ valence classes of positive Hermitian forms in 4 variables which are even integral over $\mathcal{O}$ and of determinant $16 / d^{2}$;

(b) If $s>4 k$, then $\operatorname{dim}_{c}\left(\Gamma_{\mathscr{K}}^{s}(K), 4 k\right) \geqq 1$;

(c) If $s>r$ and $r$ is not divisible by 4 , then $\left(\Gamma_{\dddot{x}}^{s}(K), r\right)=\{0\}$. 
Freitag [2] has shown that $\left(\Gamma_{\partial \mathscr{C}}^{2}(\boldsymbol{Q}(\sqrt{-1})), 4\right)$ is one-dimensional, and has constructed a generator by means of theta functions with characteristic. Define ([2], eq. (11))

$$
\Theta(Z ; \mathfrak{A}, \mathfrak{B})=\sum \exp i \pi\left(Z\left\{\mathfrak{E}+\frac{1+i}{2} \mathfrak{A}\right\}+2 \operatorname{Re}\left(\frac{1+i}{2} \mathfrak{B}^{\prime} \mathfrak{C}\right)\right),
$$

where $Z\{\mathfrak{X}\}=\overline{\mathfrak{X}}^{\prime} Z \mathfrak{X}, \mathfrak{A}, \mathfrak{B} \in Z^{2}$, and $\mathfrak{E}$ runs through $Z[\sqrt{-1}]^{2}$, and set

$$
\varphi_{4}(Z)=\sum \Theta^{4}(Z ; \mathfrak{A}, \mathfrak{B}),
$$

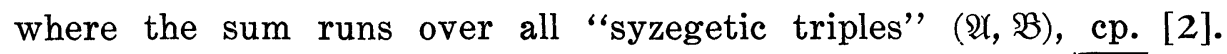
Then $\varphi_{4}$ is a Hermitian modular form of weight 4 for $\boldsymbol{Q}(\sqrt{-1})$.

THEOREM 4. Let $\mathbb{\Omega}=Z[\sqrt{-1}]^{4 \times 2}$ and $Z \in \mathbb{Z}^{2}$. There is a nonzero constant $c$ such that

$$
\varphi_{4}(Z)=c \Theta_{\mathcal{R}}(Z, I) .
$$

Indeed, the proof of Theorem 1 shows that $\Theta_{\mathscr{L}}(Z, I)$ is a Hermitian modular form of weight 4 for $\boldsymbol{Q}(\sqrt{-1})$, where $I$ is Iyanaga's Hermitian quadratic form, given by (23).

\section{REFERENCES}

1. H. Braun, Hermitian modular functions, 1, Ann. Math., 50 (1949), 827-855; 2, Ann. Math., 51 (1950), 82-104, 3, Ann. Math., 53 (1951), 143-160.

2. E. Freitag, Modulformen zweiten Grades zum rationalen und Gauss-schen Zahlkörper, Sitz. Heidelberger Akad. Wiss., Math.-naturwiss, Kl., No. 1, 1967.

3. L.J. Gerstein, Integral decomposition of Hermitian forms, Amer. J. Math., 92 (1970), 398-418.

4. K. Iyanaga, Arithmetic of special unitary groups and their symplectic representations, J. Fac. Sci. Univ. Tokyo (Section I), 15 (1968), Part 1, 35-69.

5. N. Jacobson, A note on Hermitian forms, Bull. Amer. Math. Soc., 46 (1940), 264-268.

6. H. Minkowski, Gesammelte Abhandlungen I, 1911.

7. L.E.J. Mordell, The definite quadratic forms in eight variables with determinant unity, J. Math. Pures Appl., 17 (1938), 41-46.

8. O.T. O'Meara, Introduction to Quadratic Forms, Springer-Verlag, New York 1972.

9. H. Pfeuffer, Einklassige Geschlechter total positiver quadratische Formen in totalreelen algebraischen Zahlenkörpern, J. Number Theory, 3 (1971), 371-411.

10. H.L. Resnikoff, Automorphic forms of singular weight are singular forms, Math. Ann., 215 (1975), 173-193.

11. - Theta functions for Jordan algebras, Inv. Math., 31 (1975), 87-104.

12. - The structure of spaces of singular automorphic forms, preprint 1977.

Received January 28, 1977 and in revised form September 13, 1977. Research of both authors was partially supported by NSF grant MCS76-07159.

MATHEMATISChES INSTITUT DER UNIVERSITÄT

D34 Göttingen, West Germany

AND

Department of Mathematics

UNIVERSITY OF CALIFORNIA

IRvine, CA 92717 U.S.A. 



\section{PACIFIC JOURNAL OF MATHEMATICS}

\section{EDITORS}

RICHARD ARENS (Managing Editor)

University of California

Los Angeles, CA 90024

Charles W. Curtis

University of Oregon

Eugene, OR 97403

C. C. MOORE

University of California

Berkeley, CA 94720

\section{J. DugundJI}

Department of Mathematics

University of Southern California

Los Angeles, CA 90007

R. FinN and J. Milgram

Stanford University

Stanford, CA 94305

\section{ASSOCIATE EDITORS}
E. F. BECKENBACH
B. H. NeumanN
F. WOLF
K. YosHIDA

\section{SUPPORTING INSTITUTIONS}

\author{
UNIVERSITY OF BRITISH COLUMBIA \\ CALIFORNIA INSTITUTE OF TECHNOLOGY \\ UNIVERSITY OF CALIFORNIA \\ MONTANA STATE UNIVERSITY \\ UNIVERSITY OF NEVADA, RENO \\ NEW MEXICO STATE UNIVERSITY \\ OREGON STATE UNIVERSITY \\ UNIVERSITY OF OREGON
}

\author{
UNIVERSITY OF SOUTHERN CALIFORNIA \\ STANFORD UNIVERSITY \\ UNIVERSITY OF HAWAII \\ UNIVERSITY OF TOKYO \\ UNIVERSITY OF UTAH \\ WASHINGTON STATE UNIVERSITY \\ UNIVERSITY OF WASHINGTON
}

The Supporting Institutions listed above contribute to the cost of publication of this Journal, but they are not owners or publishers and have no responsibility for its content or policies.

Mathematical papers intended for publication in the Pacific Journal of Mathematics should be in typed form or offset-reproduced, (not dittoed), double spaced with large margins. Please do not use built up fractions in the text of the manuscript. However, you may use them in the displayed equations. Underline Greek letters in red, German in green, and script in blue. The first paragraph or two must be capable of being used separately as a synopsis of the entire paper. Items of the bibliography should not be cited there unless absolutely necessary, in which case they must be identified by author and journal, rather than by item number. Manuscripts, in triplicate, may be sent to any one of the editors. Please classify according to the scheme of Math. Reviews, Index to Vol. 39. All other communications should be addressed to the managing editor, or Elaine Barth, University of California, Los Angeles, California, 90024.

50 reprints to each author are provided free for each article, only if page charges have been substantially paid. Additional copies may be obtained at cost in multiples of 50 .

The Pacific Journal of Mathematics is issued monthly as of January 1966. Regular subscription rate: $\$ 72.00$ a year (6 Vols., 12 issues). Special rate: $\$ 36.00$ a year to individual members of supporting institutions.

Subscriptions, orders for numbers issued in the last three calendar years, and changes of address should be sent to Pacific Journal of Mathematics, 103 Highland Boulevard, Berkeley, California, 94708. Older back numbers obtainable from Kraus Periodicals Co., Route 100, Millwood, NY 10546.

PUBLISHED BY PACIFIC JOURNAL OF MATHEMATICS, A NON-PROFIT CORPORATION

Printed at Kokusai Bunken Insatsusha (International Academic Printing Co., Ltd.). 8-8, 3-chome, Takadanobaba, Shinjuku-ku, Tokyo 160, Japan.

Copyright (C) 1978 by Pacific Journal of Mathematics Manufactured and first issued in Japan 


\section{Pacific Journal of Mathematics}

\section{Vol. 76, No. $2 \quad$ December, 1978}

Stephanie Brewster Brewer Taylor Alexander, Local and global convexity in complete Riemannian manifolds ...........................

Claudi Alsina i Català, On countable products and algebraic convexifications of probabilistic metric spaces ...............................

Joel David Berman and George Grätzer, Uniform representations of

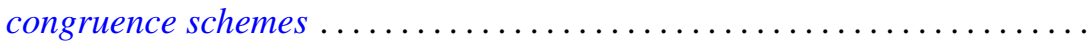

Ajit Kaur Chilana and Kenneth Allen Ross, Spectral synthesis in

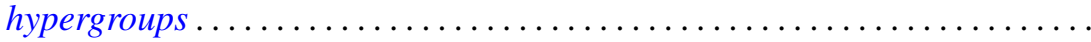

David Mordecai Cohen and Howard Leonard Resnikoff, Hermitian quadratic forms and Hermitian modular forms . .........................

Frank Rimi DeMeyer, Metabelian groups with an irreducible projective

representation of large degree .............................

Robert Ellis, The Furstenberg structure theorem .....................

Heinz W. Engl, Random fixed point theorems for multivalued mappings .......

William Andrew Ettling, On arc length sharpenings ..................

Kent Ralph Fuller and Joel K. Haack, Rings with quivers that are trees........

Kenneth R. Goodearl, Centers of regular self-injective rings ...............

John Gregory, Numerical algorithms for oscillation vectors of second order

differential equations including the Euler-Lagrange equation for

symmetric tridiagonal matrices.

Branko Grünbaum and Geoffrey Shephard, Isotoxal tilings

Myron Stanley Henry and Kenneth Leroy Wiggins, Applications of

approximation theory to differential equations with deviating

arguments

Mark Jungerman, The non-orientable genus of the n-cube .

Robert Richard Kallman, Only trivial Borel measures on $S_{\infty}$ are

quasi-invariant under automorphisms ................

Joyce Longman and Michael Rich, Scalar dependent algebras in the

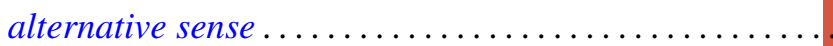

Richard A. Mollin, The Schur group of a field of characteristic zero ........ 471

David Pokrass, Some radical properties of rings with $(a, b, c)=(c, a, b) \ldots 479$

Margaret Shay and Paul Ruel Young, Characterizing the orders changed by

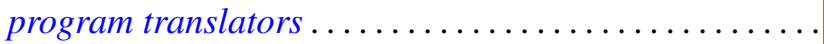

Jerrold Norman Siegel, On the structure of $B_{\infty}(F), F$ a stable space...

Surjeet Singh, (hnp)-rings over which every module admits a basic

submodule...

A. K. Snyder, Universal interpolating sets and the Nevanlinna-Pick property in

Banach spaces of functions...

Jeffrey D. Vaaler, On the metric theory of Diophantine approximation ... 